\title{
BMJ Open Patient-reported burden of dry eye disease in the UK: a cross-sectional web- based survey
}

\author{
Parwez Hossain (D) , ${ }^{1,2}$ Csaba Siffel, ${ }^{3,4}$ Corey Joseph, ${ }^{3}$ Juliette Meunier, ${ }^{5,6}$
} Jessica T. Markowitz, ${ }^{5,6}$ Reza Dana ${ }^{7,8}$

To cite: Hossain P, Siffel C, Joseph C, et al. Patient-reported burden of dry eye disease in the UK: a cross-sectional web-based survey. BMJ Open 2021;11:e039209. doi:10.1136/ bmjopen-2020-039209

- Prepublication history for this paper is available online To view these files, please visit the journal online (http://dx.doi. org/10.1136/bmjopen-2020 039209).

Received 27 April 2020 Revised 09 February 2021 Accepted 15 February 2021
Check for updates

(C) Author(s) (or their employer(s)) 2021. Re-use permitted under CC BY-NC. No commercial re-use. See rights and permissions. Published by BMJ.

For numbered affiliations see end of article.

Correspondence to Professor Parwez Hossain; parwez@soton.ac.uk

\section{ABSTRACT}

Objectives To compare sociodemographics and visionrelated quality of life $(\mathrm{Q} o \mathrm{~L})$ of individuals with or without dry eye disease (DED); and to explore the impact of DED symptom severity on visual function, activity limitations and work productivity.

Design Cross-sectional web-based survey.

Setting General UK population.

Participants Adults $\geq 18$ years with $(\mathrm{N}=1002)$ or without $(\mathrm{N}=1003)$ self-reported DED recruited through email and screened

Main outcome measures All participants completed the 25-item National Eye Institute Visual Function Questionnaire (NEI VFQ-25), with six additional questions (items A3-A8), and the EuroQol 5 dimensions 5 levels. DED participants also completed Impact of Dry Eye on Everyday Life questionnaire, 5-item Dry Eye Questionnaire and the Standardised Patient Evaluation of Eye Dryness questionnaire along with the Ocular Comfort Index, Work Productivity and Activity Impairment and the Eye Dryness Score (EDS), a Visual Analogue Scale.

Results Baseline demographic and clinical characteristics were similar in participants with versus without DED (mean age, 55.2 vs 55.0 years; $61.8 \%$ vs $61.0 \%$ women, respectively) based on recruitment targets. Scores were derived from NEI VFQ-25 using the new 28-item revised VFQ (VFQ-28R) scoring. Mean (SD) VFQ-28R scores were lower in participants with versus without DED, indicating worse functioning (activity limitations, 73.3 (12.3) vs 84.4 (12.3); socioemotional functioning, 75.3 (21.5) vs 90.3 (16.2); total score, 71.6 (12.8) vs 83.6 (12.6)). Higher percentages of problems/inability to do activities were observed among those with versus without DED. The impact of DED on visual function was worse for participants with more severe DED symptoms, as assessed by EDS. In addition, a higher EDS was associated with worse symptoms on common DED scales and a worse impact on work productivity.

Conclusions DED symptoms were associated with negative effects on visual function, activities and work productivity, whereas worse DED symptoms had a greater impact on vision-related QoL and work productivity.

\section{INTRODUCTION}

Dry eye disease (DED) is a commonly occurring ocular condition and a frequent reason for patients to seek medical eye care. ${ }^{12}$ Global

\section{Strengths and limitations of this study}

- This comprehensive study is the largest dry eye disease (DED) survey completed in the UK assessing differences in health-related and vision-related quality of life (QoL) among participants with and without DED.

- This study was a descriptive analysis based on a single-item tool to assess severity of DED symptoms (eye dryness), and this is currently undergoing psychometric validation.

- Since this was an internet-based survey, individuals without access to the internet did not have the opportunity to participate in the study.

- DED was self-reported by participants based on their symptoms, and this can be unreliable and may result in misclassification.

- As this was a cross-sectional study, we could not assess whether the vision-related QoL was low prior to a diagnosis of DED and whether vision-related QoL changed over time.

DED prevalence is estimated to range from $5 \%$ to $50 \%,{ }^{2}$ with estimates in Europe ranging from $10 \%$ to $30 \% .^{3-5}$ In a female cohort in the UK ( $\mathrm{n}=3824$; mean age, 57.1 years $)$, the overall prevalence of DED was $9.6 \%$ (defined as diagnosis by a clinician and use of artificial tears) and the prevalence of reported symptoms of DED in the previous 3 months was $20.8 \%{ }^{4}$ Prevalence of DED in the UK is higher in women compared with men and increases with age, with the majority of individuals with DED aged $>50$ years. ${ }^{46}$

DED is a major cause for prescribing in primary medical care in the National Health Service in England. In 2014, over 6.4 million prescription items for DED (ie, artificial tears, ocular lubricants and astringents) were dispensed in the community at a cost of more than $£ 27$ million to the National Health Service. $^{7}$

DED can impact health-related and vision-related quality of life (QoL), with affected patients showing greater functional 


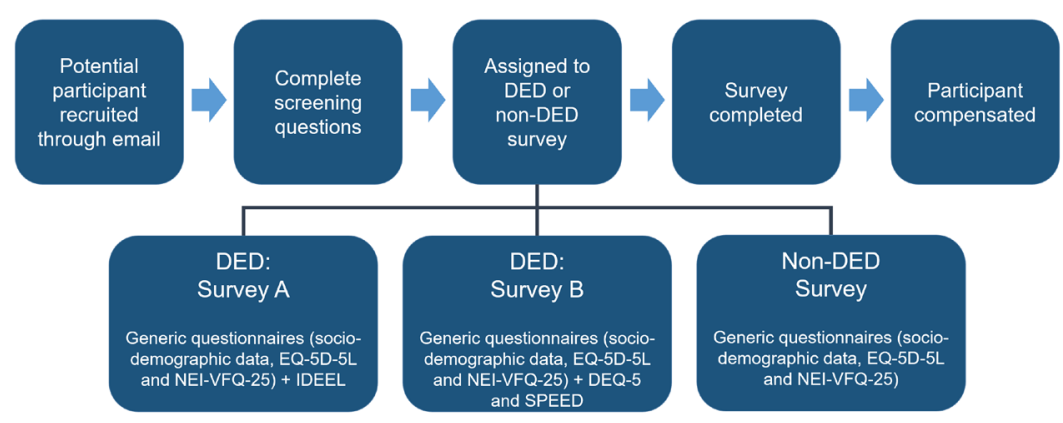

Figure 1 Study flow. DED, dry eye disease; DEQ-5, 5-item Dry Eye Questionnaire; EQ-5D-5L, EuroQol 5 dimensions 5 levels; IDEEL, Impact of Dry Eye on Everyday Life; NEI VFQ-25, 25-item National Eye Institute Visual Function Questionnaire; SPEED, Standardised Patient Evaluation of Eye Dryness.

impairment associated with physical fatigue, pain, depression, total symptom burden and QoL. ${ }^{8}$ Moreover, patients are more likely to experience problems with reading, television and computer use, driving and performing professional work. ${ }^{9}$ In addition, DED was associated with approximately $30 \%$ impairment in workplace performance, work productivity and non-job-related activities in a prospective cross-sectional study. ${ }^{10}$

In a systematic literature search supplemented with information from interviews of ophthalmologists, the annual healthcare costs for 1000 patients with DED who were managed by ophthalmologists in the UK were estimated to be US $\$ 1.10$ million (2003/2004 prices), with almost one-half of the total attributed to prescription drug costs. Some patients suffering from DED self-treat their symptoms with over-the-counter artificial tears or are treated by general practitioners/optometrists/pharmacists; thus, annual costs could be higher as only reimbursed treatments prescribed by ophthalmologists were captured in this estimate. ${ }^{11}$

Given the influence of DED on QoL and healthcare costs, and the minimal published data in the UK, this study was performed to document the burden of DED among adults in the UK. We conducted an online survey and compared the demographics and overall QoL between those with and without DED; evaluated the severity of symptoms and their impact on vision-related QoL and work productivity; assessed the behaviours for seeking care and adhering to treatment and assessed the use of medications for DED.

\section{METHODS}

This was a cross-sectional, web-based survey of individuals in the UK with and without DED. Survey participants were $\geq 18$ years of age; resided in the UK at the time of survey completion; spoke, read and understood English and had a self-reported diagnosis of DED or symptoms of DED (for those in the group with DED).
Figure 1 shows an overview of the study flow. Approximately 1000 participants with self-reported DED and approximately 1000 participants without DED were recruited via email from panels of patients who previously agreed to participate in online research about healthcarerelated issues. All participants consented electronically before completing the survey.

Screening included questions about gender and age to fulfil recruitment targets, as well as one multiple-choice question regarding specific medical conditions, including DED. Participants who indicated they did not have a DED diagnosis were asked follow-up questions to determine if they had experienced symptoms of DED (eye discomfort, including feelings of dryness, grittiness or soreness; burning sensation; feeling like something is in the eye; eyelids that stick together on awakening or temporary blurred vision, which usually improves when blinking). If they indicated a diagnosis of DED or had two or more of the symptoms, they were included in the group with DED. If not, they were invited to complete the non-DED survey.

Recruited participants received an invitation for the survey, which included a unique link to complete the online survey using Confirmit Horizon, a web-based platform. All participants with DED were assigned one of two approximately 45-minute surveys (survey A or survey B) on an alternating basis to reduce participant completion burden, since several questionnaires had to be completed. Participants without DED completed the non-DED survey. All survey participants completed a sociodemographic form (including racial designation, level of education, working status and description of daily living environment); a medical history; the EuroQol 5 dimensions 5 levels (EQ-5D-5L) and the 25-item National Eye Institute Visual Function Questionnaire (NEI VFQ-25) assessing visual function, with six additional items (items A3-A8). In the NEI VFQ-25, an appendix with 14 optional additional items exists (VFQ-39), which users can add to a specific subscale. Six of these items were of particular interest for this survey, including 'near vision' and 'distance vision'. 
Table 1 Outcome variables

\begin{tabular}{|c|c|c|}
\hline Assessment & Use & Score \\
\hline VFQ-28R ${ }^{18}$ & $\begin{array}{l}\text { A scoring algorithm developed for the NEI VFQ-25 for } \\
\text { patients with chronic dry eye that assesses vision- } \\
\text { related quality of life } \\
\text { Measures activity limitation (near activities, distance } \\
\text { activities, peripheral vision, colour vision, driving, } \\
\text { social functioning and six additional questions) and } \\
\text { socioemotional impact (ocular pain, mental health, role } \\
\text { difficulties and dependency) }\end{array}$ & $\begin{array}{l}\text { Scores range from } 0 \text { to } 100 \text {; a lower score indicates } \\
\text { more activity limitations and socioemotional impact }\end{array}$ \\
\hline $\mathrm{OCl}^{22}$ & $\begin{array}{l}\text { Measures ocular surface irritation over the past week } \\
\text { 12-item questionnaire with six typical symptoms } \\
\text { (dryness, grittiness, stinging, tiredness, pain and } \\
\text { itchiness) and subquestions for each symptom to } \\
\text { identify the frequency and intensity of DED }\end{array}$ & $\begin{array}{l}\text { Each item scored on a scale of } 0 \text { (no symptoms) to } 6 \\
\text { (most frequent/most severe) } \\
\text { Total score: } 0-72\end{array}$ \\
\hline IDEEL $^{24}$ & $\begin{array}{l}\text { Assesses the impact of dry eye symptoms on aspects of } \\
\text { a patient's daily life (ie, emotional impact, daily activities, } \\
\text { impact of work, satisfaction with treatment effectiveness, } \\
\text { symptom bother, treatment-related bother) }\end{array}$ & $\begin{array}{l}\text { Scores on each dimension range from } 0 \text { to 100; higher } \\
\text { scores on the Dry Eye Impact on Daily Life module (ie, } \\
\text { emotional impact, daily activities, and impact of work) } \\
\text { indicate less impact; higher scores for symptom bother } \\
\text { indicate greater bother due to symptoms; higher scores } \\
\text { for satisfaction with treatment effectiveness indicate } \\
\text { greater satisfaction; higher scores with treatment- } \\
\text { related bother indicate less treatment-related bother }\end{array}$ \\
\hline
\end{tabular}

DED, dry eye disease; DEQ-5, 5-item Dry Eye Questionnaire; EQ-5D-5L, EuroQol 5 dimensions 5 levels; IDEEL, Impact of Dry Eye on Everyday Life; $\mathrm{OCl}$, Ocular Comfort Index; SPEED, Standardised Patient Evaluation of Eye Dryness; NEI VFQ-25, 25-item National Eye Institute Visual Function Questionnaire; VFQ-28R, 28 item revised Visual Function Questionnaire; WPAI, Work Productivity and Activity Impairment.

These items were part of the development of the NEI VFQ and, thus, have been validated. ${ }^{12}$ The DED survey included the following assessments: symptom severity/ frequency Visual Analogue Scale (VAS), Ocular Comfort Index (OCI), Work Productivity and Activity Impairment (WPAI) questionnaire, treatment and resource utilisation, treatment satisfaction and treatment compliance and discontinuation. In addition, participants assigned to survey A completed the Impact of Dry Eye on Everyday Life (IDEEL) questionnaire, and those assigned to survey B completed the 5-item Dry Eye Questionnaire (DEQ-5) and the Standardised Patient Evaluation of Eye Dryness (SPEED) questionnaire. Participants had to complete all questions; missing data were not allowed.

Participants were compensated by the marketing agency that recruited them and fielded the survey; the costs were reimbursed by the study sponsor. Participants were enrolled in a panel where they could earn 'panel points' for participation in surveys and were compensated with these 'panel points', which also varied based on participant's tenure in the panel.

Participants with DED were grouped according to DED severity using the Eye Dryness Score (EDS) VAS (0-100; $0=$ no discomfort, $100=$ maximal discomfort), which was developed in conjunction with clinical trials conducted in patients with DED. Group 1 was defined as participants with DED and EDS $<40$; group 2, $40 \leq \mathrm{EDS}<60$ and group $3, \mathrm{EDS} \geq 60$. Eye dryness is an important symptom of DED that many participants felt was the most relevant. ${ }^{13} 14$ The EDS identifies the severity of this essential part of DED. ${ }^{15-17}$ Scores from all questionnaires were calculated for each DED severity group; scores from the NEI VFQ-25 were calculated using the new 28-item revised VFQ (VFQ28R) scoring. ${ }^{18}$

Table 1 includes a brief description of each of the outcome variables included in the survey. 


\begin{tabular}{|c|c|c|}
\hline Demographics & $\begin{array}{l}\text { Participants with } \\
\text { DED* } \\
(\mathrm{N}=1002)\end{array}$ & $\begin{array}{l}\text { Participants } \\
\text { without DED } \\
(\mathrm{N}=1003)\end{array}$ \\
\hline \multicolumn{3}{|l|}{ Age, years } \\
\hline Mean (SD) & $55.2(15.2)$ & $55.0(15.7)$ \\
\hline Range & $18-84$ & $18-99$ \\
\hline Woman, n (\%) & $619(61.8)$ & $612(61.0)$ \\
\hline \multicolumn{3}{|l|}{$\begin{array}{l}\text { Racial/ethnic designation, } \\
\% \dagger\end{array}$} \\
\hline White British & 89.8 & 90.6 \\
\hline Any other white & 4.0 & 4.4 \\
\hline Asian/Asian British & 4.1 & 3.1 \\
\hline African/Caribbean/black & 1.2 & 0.8 \\
\hline Other ethnic group & 0.8 & 1.2 \\
\hline Prefer not to answer & 1.1 & 0.5 \\
\hline
\end{tabular}

*Participants with DED who completed survey $A, n=500$; participants with DED who completed survey $B, n=502$.

†Several answers were possible; thus, the total percentage is greater than $100 \%$.

DED, dry eye disease.

Identifiable participant data were anonymised prior to analysis. Analyses included descriptions of the following: (1) sociodemographic, diagnosis and medical history data for participants with and without DED, (2) VFQ28R items with domain scores and EQ-5D-5L for participants with and without DED and (3) overall DED-related burden items and scores from surveys $\mathrm{A}$ and $\mathrm{B}$ and by EDS severity. No hypothesis was formulated; therefore, no inferential statistics were performed.

\section{Patient and public involvement}

This research was done without patient involvement. Patients were not invited to comment on the study design and were not consulted to develop patient relevant outcomes or interpret the results. Patients were not invited to contribute to the writing or editing of this document for readability or accuracy. There are no plans to disseminate the results of the research directly to study participants.

\section{RESULTS}

\section{Demographics}

Baseline demographic characteristics of participants with DED ( $\mathrm{N}=1002$; survey $A, n=500$; survey $\mathrm{B}, \mathrm{n}=502$ ) and without DED ( $\mathrm{N}=1003)$ were well balanced based on recruitment targets (table 2). There were 761 participants who started the survey but did not complete it.

More than $80 \%$ of survey respondents in either cohort reported the use of digital screens or daily activities such as reading. Other daily environmental factors associated with DED were reported more frequently by participants with versus without DED (ie, air conditioning/recirculated air, $25.1 \%$ vs $15.4 \%$; wind or moving air, $32.3 \%$ vs $12.3 \%$; forced air/heat, $17.6 \%$ vs $8.4 \%$; polluted air,
$13.5 \%$ vs $7.6 \%$; low humidity, $15.0 \%$ vs $5.8 \%$ ). The most commonly reported medical conditions from the screener form in participants with DED (other than DED) were arthritis $(38.1 \%)$, hearing loss $(24.5 \%)$, irritable bowel disease $(23.2 \%)$ and asthma (16.9\%). The most common medical conditions in participants without DED were arthritis $(20.9 \%)$, asthma (10.4\%), hearing loss (9.4\%) and irritable bowel disease $(8.9 \%)$.

Participants with DED were grouped according to severity based on EDS VAS: group $1(\mathrm{EDS}<40), \mathrm{n}=534$

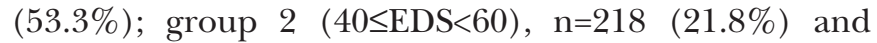
group 3 (EDS $\geq 60), n=250$ (25.0\%). Table 3 includes diagnosis and medical history data for participants with a selfreported diagnosis of DED. Participants with more severe DED were more likely to be diagnosed and treated by a healthcare professional $(44.8 \%$ in group 1 vs $75.2 \%$ in group 3). Approximately half of the healthcare professionals were ophthalmologists (eye doctors), and the rest were primary care doctors or other kind of healthcare professionals.

\section{Comparison of scores between participants with and without DED}

Mean (SD) VFQ-28R activity limitations scores, socioemotional functioning scores and total scores reflect good visual functioning in participants without DED, while participants with DED had worse visual functioning (figure 2).

The responses on the EQ-5D-5L showed higher proportions of participants with DED who had problems with mobility, self-care and usual activities; more pain and/ or discomfort and more anxiety and/or depression (figure 3).

\section{Vision-related QoL, health status and work productivity of participants with DED}

The highest mean (SD) VAS scores for 24-hour DED symptoms were for eye dryness $(37.9$ (29.3)), tired eyes (37.6 (29.2)) and eye discomfort (32.6 (28.2)). For the scales other than the EDS, mean VAS scores for DED symptoms increased as the severity assessed by the EDS increased.

Symptom severity determined by EDS was associated with severity on common DED instruments, such as the OCI. The mean (SD) overall OCI score for participants with DED was 30.3 (14.1) and increased as the severity of DED increased, as shown in figure 4 . The same trend was observed with DEQ-5 and SPEED. Mean (SD) DEQ-5 total score was 8.9 (3.6) for group 1, 11.1 (2.7) for group 2 and 13.4 (3.5) for group 3, indicating a higher severity of DED with increasing DEQ-5 scores. For SPEED, the total mean (SD) scores for participants with DED were 9.6 (5.2) in group 1, 11.9 (5.6) in group 2 and 15.6 (5.1) in group 3 . The increasing mean score for SPEED indicated more frequent and/or worsening symptoms as DED severity increased.

All mean (SD) VFQ-28R scores (activity limitations, socioemotional functioning and total) globally decreased 
as the severity of DED increased, indicating worsening visual function as DED severity increased (figure 5).

Mean (SD) EQ-5D index scores decreased as DED severity increased, reflecting worsening health status in participants with DED and the highest EDS (group 1, 0.77 (0.23); group 2, $0.70(0.24)$; group 3, $0.67(0.26))$. In addition, the percentage of participants reporting problems with each EQ-5D item rose with increasing DED severity (figure 6).

WPAI scores increased with worsening DED severity for 'percent activity impairment due to problem'. However, mean scores for 'percent impairment while working due to a problem', 'percent overall work impairment due to problem' and 'percent work time missed due to problem' increased from group 1 to group 2, but the mean scores were similar or decreased from group 2 to group 3 (figure 7).

For the IDEEL scores of participants with DED stratified by severity group, greater emotional impact, greater impact on daily activities, greater symptom bother and lower satisfaction with treatment effectiveness were associated with increasing EDS scores. In addition, a greater impact on work and more treatment-related bother were observed with a higher DED severity, though the difference between groups 2 and 3 was not as well defined.

\section{DISCUSSION}

Previous studies have shown an association between DED and reduced health-related and vision-related QoL. ${ }^{9} 1920$ The present study demonstrates that this association is consistent in a population of participants with DED from the UK. Dry eye symptoms were related to a negative impact on daily activities, socioemotional functioning and general health status among participants from a large general population sample in the UK. Increased DED symptom severity was associated with a greater impact on activities, socioemotional functioning and work productivity.

While causal associations cannot be elucidated in this cross-sectional study, problems with mobility, as assessed by the EQ-5D-5L, occurred more often in participants with DED than in participants without DED (36\% of participants reported having either slight, moderate,

Table 3 Participants with DED: diagnosis and medical history

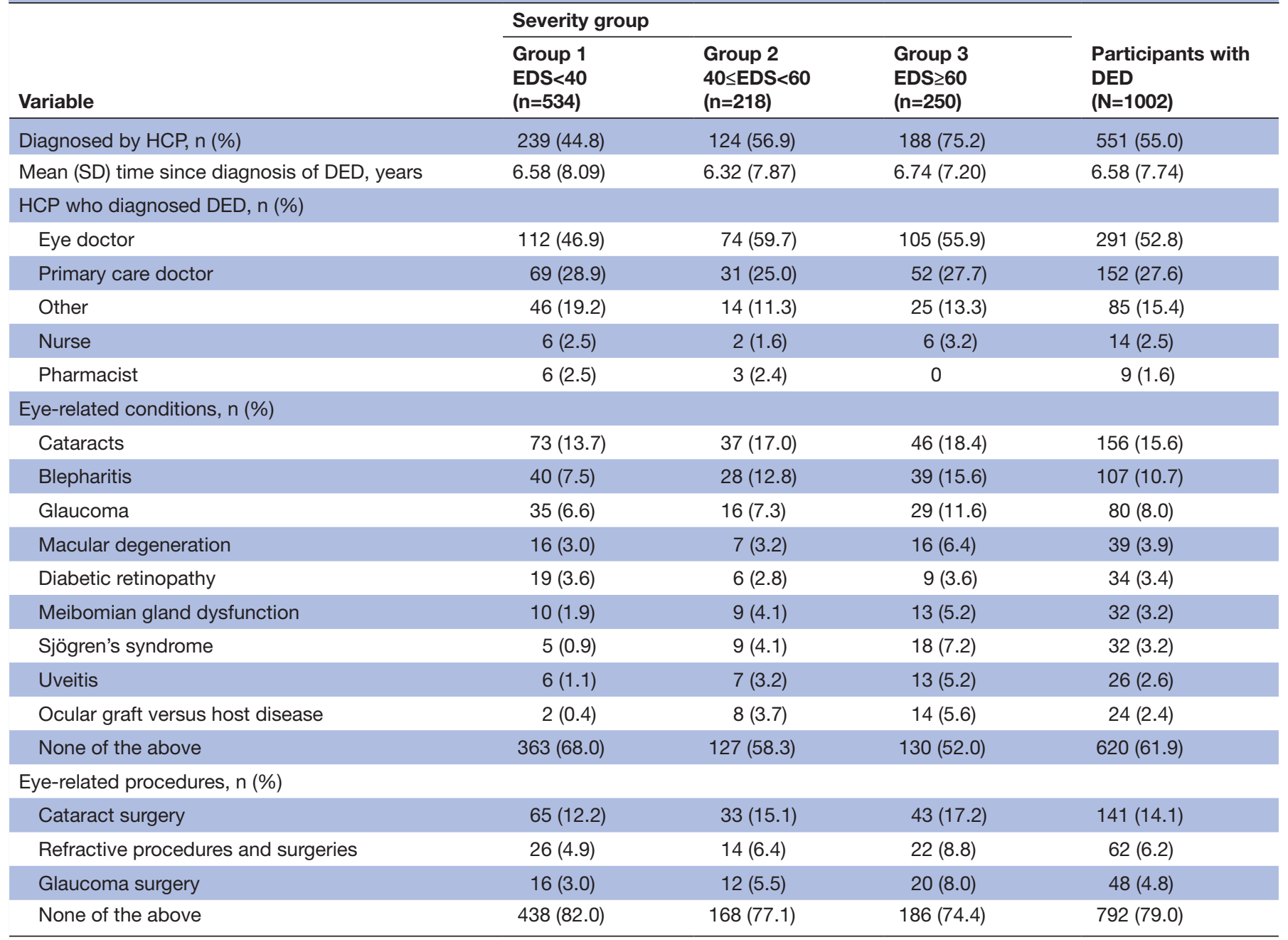

DED, dry eye disease; EDS, Eye Dryness Score; HCP, healthcare professional. 


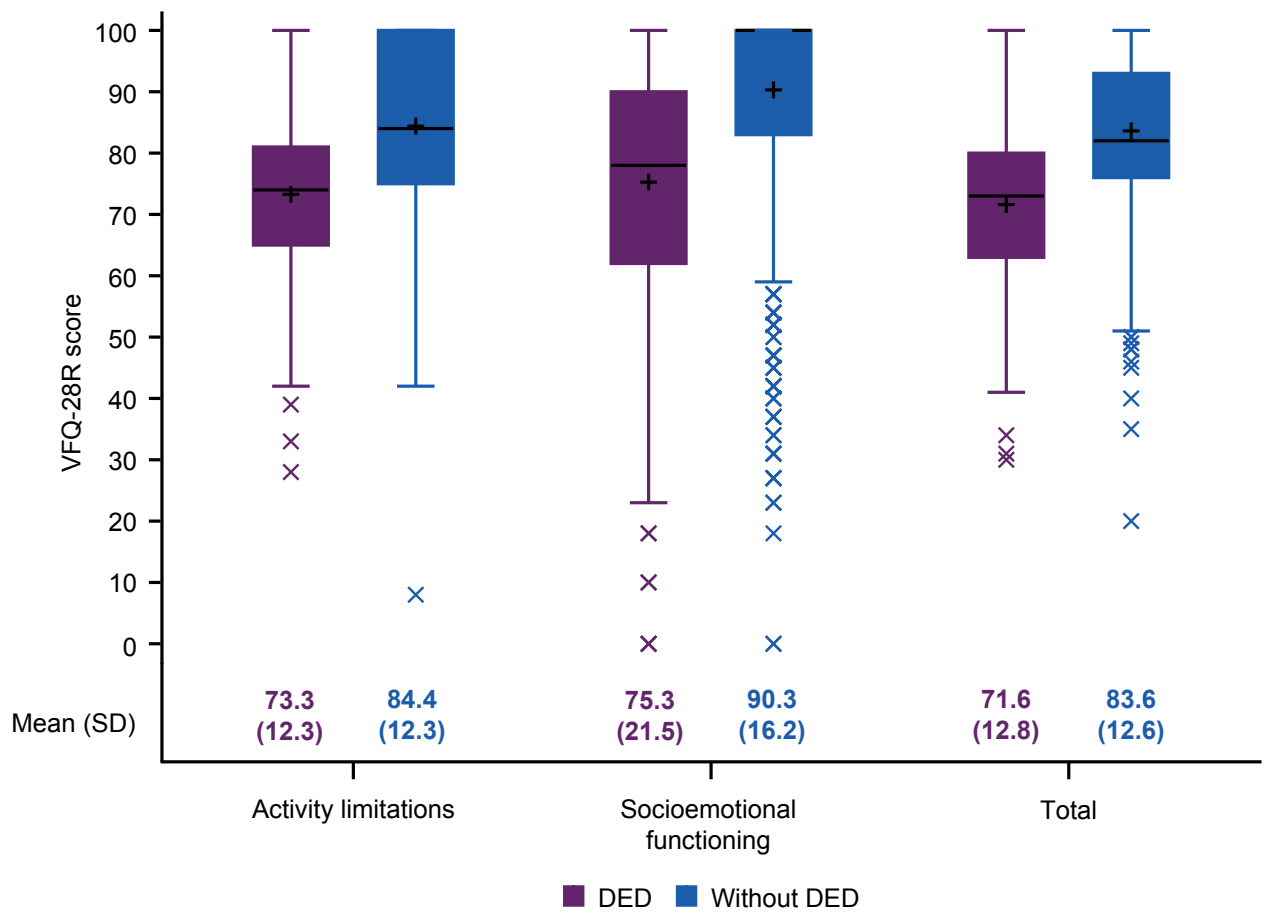

Figure 2 VFQ-28R scores in participants with versus without DED. Box for each score: IQR (Q1-Q3); +, mean; -, median; bottom and top bars, observed minimum and maximum values; $\times$, outliers (ie, values that are outside the distance of 1.5 times the IQR from Q1 or Q3). DED, dry eye disease; Q, quarter; VFQ-28R, 28-item revised Visual Function Questionnaire.

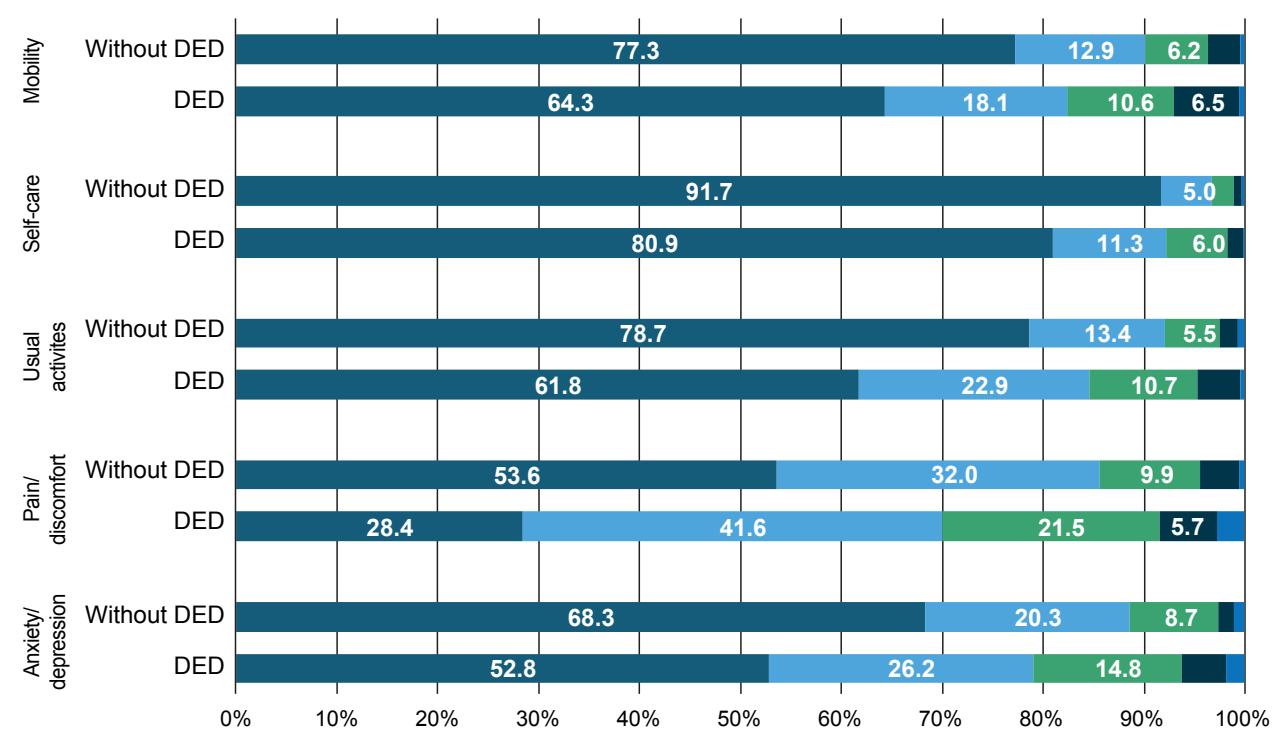

- No problems/no pain or discomfort/not anxious or depresssed

- Slight problems/slight pain or discomfort/slightly anxious or depressed

- Moderate problems/moderate pain or discomfort/moderately anxious or depressed

- Severe problems/severe pain or discomfort/severely anxious or depressed

- Unable/extreme pain or discomfort/extremely anxious or depressed

Figure 3 Description of answers to EQ-5D-5L items among participants with and without DED. Percentages not shown on figure were $<5 \%$. DED, dry eye disease; EQ-5D-5L, EuroQol 5 dimensions 5 levels.

severe problems or being unable to move vs $23 \%$ of participants without DED). In addition, more problems with self-care (19\% vs $8 \%)$, usual activities (38\% vs $21 \%$ ) and anxiety/depression ( $47 \%$ vs $32 \%$ ) were reported in participants with DED than in participants without DED. This difference could be explained by the presence of DED; however, the higher prevalence of comorbidities in participants with DED could be a contributing factor. The 


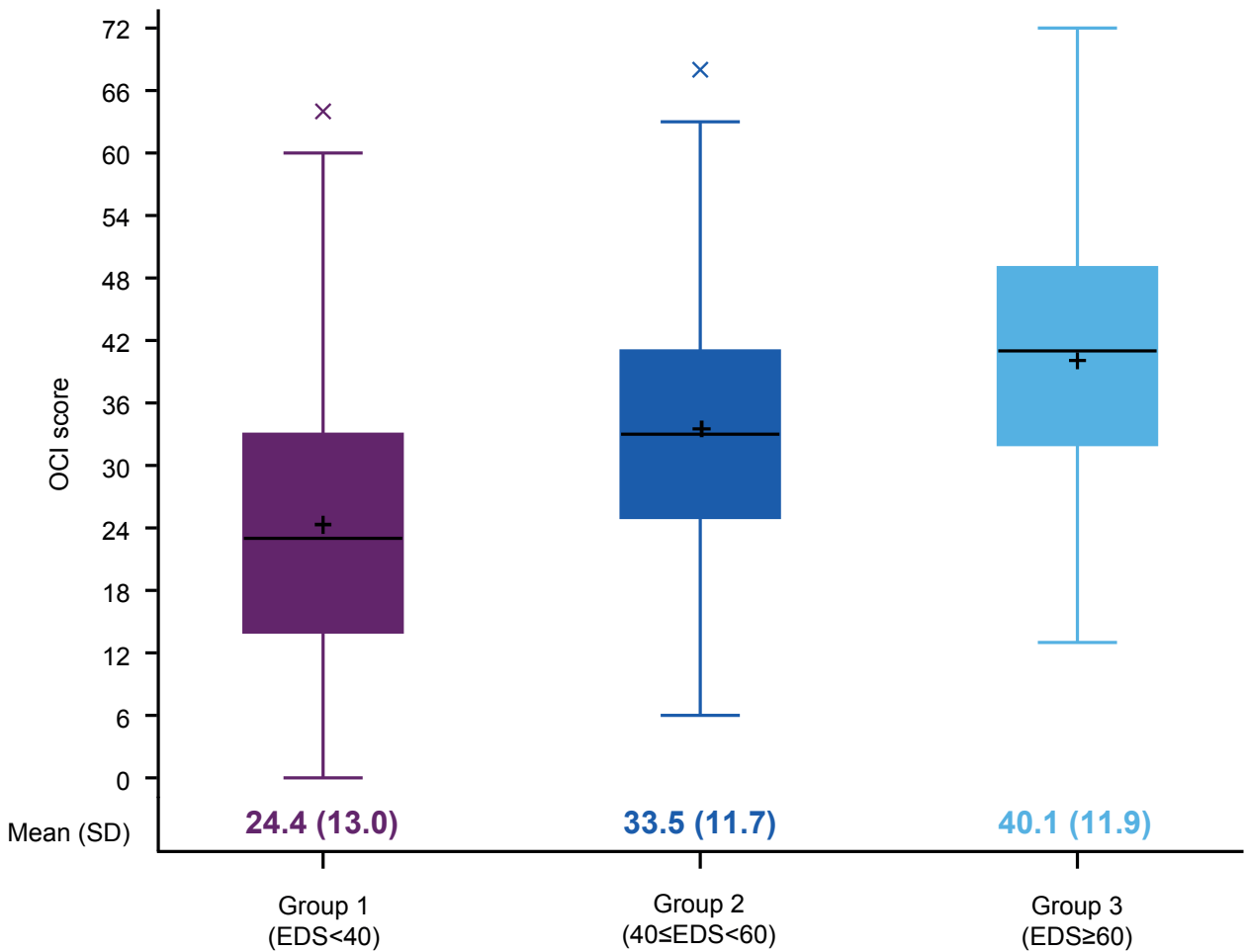

Figure $4 \mathrm{OCl}$ scores by severity group. Box for each score: IQR (Q1-Q3); +, mean; -, median; bottom and top bars, observed minimum and maximum values; $\times$, outliers (ie, values that are outside the distance of 1.5 times the IQR from Q1 or Q3). EDS, Eye Dryness Score; OCl, Ocular Comfort Index.

ocular comorbidity with the greatest difference between participants with and without DED was pain/discomfort: $72 \%$ of participants with DED reported some level of discomfort, while only $46 \%$ of participants without DED reported the same. Since the EQ-5D-5L is a measure of overall health status, it is very likely that participants with

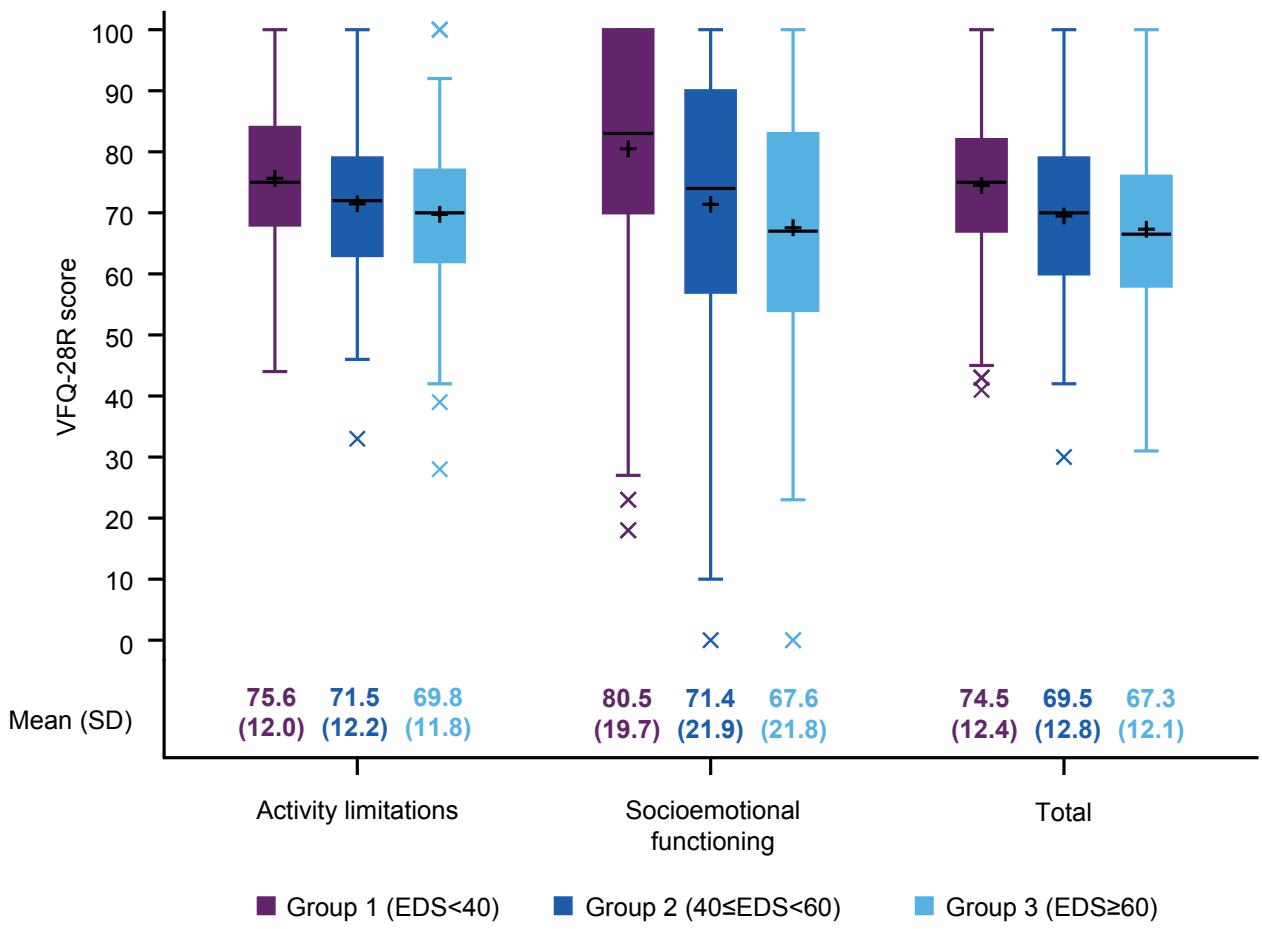

Figure 5 VFQ-28R scores by severity group. Box for each score: IQR (Q1-Q3); +, mean; -, median; bottom and top bars, observed minimum and maximum values; $\times$, outliers (ie, values that are outside the distance of 1.5 times the IQR from Q1 or Q3). EDS, Eye Dryness Score; Q, quarter; VFQ-28R, 28-item revised Visual Function Questionnaire. 

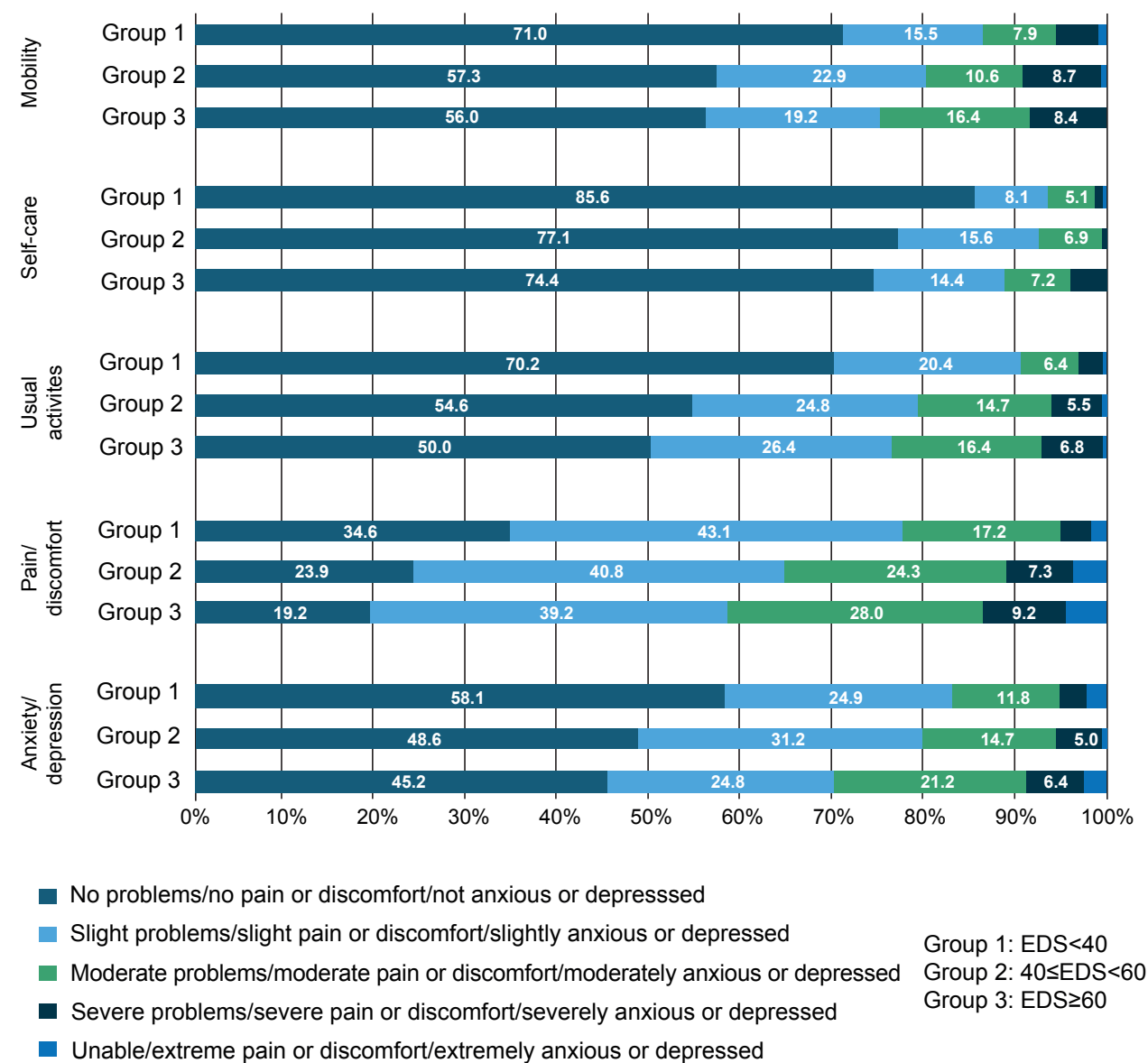

Figure 6 Description of answers to EQ-5D-5L items by severity group. Percentages not shown on figure were $<5 \%$. EDS, Eye Dryness Score; EQ-5D-5L, EuroQol 5 dimensions 5 levels.

comorbidities had lower scores (indicating lower health status) than participants without comorbidities. More sophisticated analyses would be required to answer this question. Overall health status (EQ-5D index score) was lower for participants with DED, indicating that this cohort with DED experienced reduced health status compared with those without DED.

Due to the sampling techniques used to enrol participants into the study, baseline characteristics of participants with and without DED were comparable on average. There were similar proportions of men and women, similar mean ages, digital screen use and activities such as reading, irrespective of disease status. However, other environmental factors were more frequently reported by participants with DED (air conditioning, forced air, low humidity and polluted air); these factors could contribute to the development of DED or could be noticed more by those with DED. ${ }^{2}$ Comorbidities were more prevalent in participants with DED than in those without (eg, arthritis, $38 \%$ vs $21 \%$; hearing loss, $24 \%$ vs $9 \%$ ). While causal associations cannot be drawn in a cross-sectional study, the presence of DED appeared to impact negatively on participants' health-related and vision-related QoL.

Participants were categorised into groups 1-3 according to their 24-hour EDS single-item VAS (range, 0-100), with a higher score indicating a higher severity. Those in group 3 with the highest EDS VAS had the highest level of discomfort for all symptoms (eg, eye discomfort, tired eyes, itching eyes) related to DED and a greater impact on activities and socioemotional functioning (assessed by VFQ-28R). Similarly, the mean OCI score also increased in participants with DED as severity increased, with greater discomfort observed in the eyes of participants with a higher EDS. A similar trend was seen for other questionnaires. Participants with higher severity as assessed by EDS had a higher severity and frequency of symptoms as assessed by mean DEQ-5 scores. Participants with higher severity also had higher mean SPEED scores, indicating more frequent and/or more severe symptoms.

Mean scores for WPAI globally increased as the severity increased. This reflects a higher impact of DED on activity impairment as the EDS increased. For impairment while working, overall work impairment and time missed from work, the mean scores were slightly lower in severity group 3 versus group 2. This could be due to a limitation in the definition of the EDS severity categories, or there is only a slight association between DED and work productivity and activity impairment.

The results of this study suggest the usefulness of the EDS as a single score to assess the severity of dry eye symptoms in individuals with DED. Compared with other common DED symptom scales, a higher EDS (indicating 


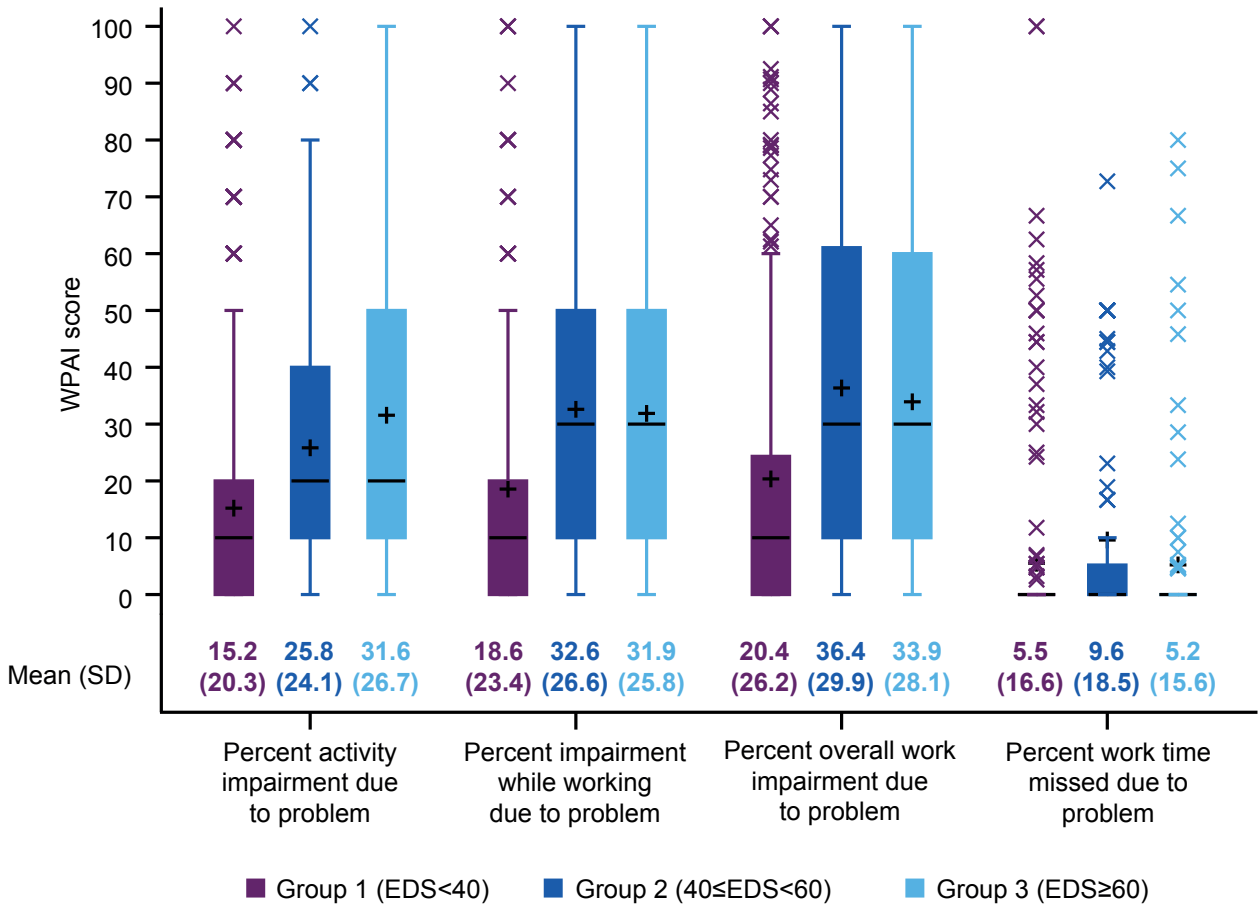

Figure 7 WPAl subscale scores by severity group. Box for each score: IQR (Q1-Q3); +, mean; -, median; bottom and top bars, observed minimum and maximum values; $\times$, outliers (ie, values that are outside the distance of 1.5 times the IQR from Q1 or Q3). EDS, Eye Dryness Score; Q, quarter; WPAl, Work Productivity and Activity Impairment.

more discomfort) was associated with worse symptoms on the OCI, DEQ-5 and SPEED. Additional analyses should be performed to confirm the reliability of the EDS to discriminate between different levels of DED severity. No inferential statistics were performed due to the nature of this study; hence, future studies could benefit from applying statistical tests to validate the associations and strengths of associations of the variables studied.

\section{Strengths and limitations of this study}

This comprehensive study is the largest DED survey completed in the UK assessing differences in healthrelated and vision-related QoL among participants with and without DED. However, the findings from this study have some limitations. First, this study was a descriptive analysis based on a single-item tool to assess severity of DED symptoms (eye dryness), and this is currently undergoing psychometric validation. Second, since this was an internet-based survey, individuals without access to the internet did not have the opportunity to participate in the study. Third, DED was self-reported by participants based on their symptoms, and this can be unreliable and may result in misclassification. Fourth, DED was diagnosed by a healthcare professional in approximately $50 \%$ of the participants included in DED groups 1 and 2, and $75 \%$ in group 3, and only half of the healthcare professionals were ophthalmologists, which may impact the accuracy of DED diagnosis and classification. Lastly, as this was a cross-sectional study, we could not assess whether the vision-related QoL was low prior to a diagnosis of DED and whether vision-related QoL changed over time.

\section{CONCLUSIONS}

This analysis provides useful information regarding participants' perspectives on the burden of DED in the UK. DED symptoms were associated with negative impacts to socioemotional functioning, vision-related QoL function, daily activities and work productivity. Participants with worse DED symptoms had a greater decline in socioemotional functioning, vision-related QoL, daily activities and work productivity. Symptom severity determined by EDS was associated with severity on common DED instruments, such as OCI, DEQ-5, and SPEED, suggesting that the EDS may be a useful single-item questionnaire for assessing severity of symptoms in individuals with DED.

\section{Author affiliations}

${ }^{1}$ Clinical Experimental Sciences, Faculty of Medicine, University of Southampton, Southampton, UK

${ }^{2}$ Eye Unit, Southampton General Hospital, University Hospitals Southampton NHS Foundation Trust, Southampton, UK

${ }^{3}$ Global Evidence and Outcomes, Takeda, Lexington, Massachusetts, USA

${ }^{4}$ Department of Interdisciplinary Health Sciences, College of Allied Health Sciences, Augusta University, Augusta, Georgia, USA

${ }^{5}$ Patient Centered Outcomes, Modus Outcomes, Lyon, France

${ }^{6}$ Patient Centered Outcomes, Modus Outcomes, Cambridge, Massachusetts, USA ${ }^{7}$ Cornea and Refractive Surgery Service, Massachusetts Eye and Ear, Boston, Massachusetts, USA

${ }^{8}$ Department of Ophthalmology, Harvard Medical School, Boston, Massachusetts, USA 
Acknowledgements We acknowledge Ipek Özer Stillman, from Takeda, for her assistance with survey conceptualisation and development, interpretation of the data and study operations; and Patrick Marquis from Modus Outcomes for his assistance with survey conceptualisation and development. We thank Lisa 0'Brien, PharmD, of Excel Scientific Solutions, who provided medical writing assistance, supported by Takeda and Novartis.

Contributors All authors had full access to all the data in the study and take responsibility for the integrity of the data and the accuracy of the data analysis. Concept and design: PH, CS, CJ, JTM, JM and RD. Acquisition, analysis or interpretation of data: PH, CS, CJ, JTM, JM and RD. Drafting of the manuscript and review: PH, CS, CJ, JTM, JM and RD. All authors approved the final manuscript as submitted and agree to be accountable for all aspects of the work. The corresponding author attests that all listed authors meet authorship criteria and that no others meeting the criteria have been omitted.

Funding This study was supported by Takeda (Award/ Grant number: N/A) and Novartis (Award/ Grant number: N/A)

Competing interests $\mathrm{PH}$ is a consultant for Dompé, Santen and Shire*. CS is an employee of and owns stock/stock options in Takeda. CJ is an employee of and owns stock/stock options in Takeda. JM is an employee of Modus Outcomes and has been a consultant for Shire* on this study. JTM is an employee of Modus Outcomes and has been a consultant for Shire ${ }^{\star}$ on this study. RD is a consultant for Aldeyra, Dompé, GSK, Kala and Shire*, holds equity in Aramis Biosciences and Claris Biotherapeutics and reports receiving grant support from Allergan and the National Eye Institute. *A Takeda company.

Patient and public involvement Patients and/or the public were not involved in the design, or conduct, or reporting or dissemination plans of this research.

Patient consent for publication Not required.

Ethics approval The study was approved by the New England Independent Review Board.

Provenance and peer review Not commissioned; externally peer reviewed.

Data availability statement Data are available upon reasonable request. All data relevant to the study are included in the article or uploaded as supplementary information. We confirm that the data generated by our research supports our current article. We confirm that we have included all our generated data in this manuscript. Novartis is committed to sharing with qualified external researchers, access to patient level data and supporting clinical documents from eligible studies. These requests are reviewed and approved by an independent review panel on the basis of scientific merit. All data provided are anonymised to respect the privacy of patients who have participated in the online surveys in line with applicable laws and regulations. The authors confirm they had no special access or privileges that other researchers would not have. Unfortunately, we are unable to provide copies of the survey as the questions asked were from copyrighted questionnaires.

Open access This is an open access article distributed in accordance with the Creative Commons Attribution Non Commercial (CC BY-NC 4.0) license, which permits others to distribute, remix, adapt, build upon this work non-commercially, and license their derivative works on different terms, provided the original work is properly cited, appropriate credit is given, any changes made indicated, and the use is non-commercial. See: http://creativecommons.org/licenses/by-nc/4.0/.

\section{ORCID iD}

Parwez Hossain http://orcid.org/0000-0002-3131-2395

\section{REFERENCES}

1 Bradley JL, Özer Stillman I, Pivneva I, et al. Dry eye disease ranking among common reasons for seeking eye care in a large US claims database. Clin Ophthalmol 2019;13:225-32.

2 Stapleton F, Alves M, Bunya VY, et al. TFOS DEWS II epidemiology report. Ocul Surf 2017;15:334-65.

3 Malet F, Le Goff M, Colin J, et al. Dry eye disease in French elderly subjects: the Alienor Study. Acta Ophthalmol 2014;92:e429-36.
4 Vehof J, Kozareva D, Hysi PG, et al. Prevalence and risk factors of dry eye disease in a British female cohort. Br J Ophthalmol 2014;98:1712-7.

5 Viso E, Rodriguez-Ares MT, Gude F. Prevalence of and associated factors for dry eye in a Spanish adult population (the Salnes Eye Study). Ophthalmic Epidemiol 2009;16:15-21.

6 Habib N, Venerus A, Grimson F, et al.. Characteristics and treatment patterns of patients with dry eye disease (DED) in secondary eye-care settings in the United Kingdom. Royal College of Ophthalmologists Annual Congress, Glasgow, UK, 2019.

7 The management of dry eye. Drug Ther Bull 2016;54:9-12.

8 Hackett KL, Newton JL, Frith J, et al. Impaired functional status in primary Sjögren's syndrome. Arthritis Care Res 2012;64:1760-4.

9 Miljanović B, Dana R, Sullivan DA, et al. Impact of dry eye syndrome on vision-related quality of life. Am J Ophthalmol 2007;143:409-15.

10 Nichols KK, Bacharach J, Holland E, et al. Impact of dry eye disease on work productivity, and patients' satisfaction with over-the-counter dry eye treatments. Invest Ophthalmol Vis Sci 2016;57:2975-82.

11 Clegg JP, Guest JF, Lehman A, et al. The annual cost of dry eye syndrome in France, Germany, Italy, Spain, Sweden and the United Kingdom among patients managed by ophthalmologists. Ophthalmic Epidemiol 2006;13:263-74.

12 Mangione CM. NEI VFQ-25 scoring algorithm. Rockville, MD: National Eye Institute, 2000. https://www.nei.nih.gov/sites/default/ files/2019-06/manual_cm2000.pdf

13 Begley CG, Chalmers RL, Abetz L, et al. The relationship between habitual patient-reported symptoms and clinical signs among patients with dry eye of varying severity. Invest Ophthalmol Vis Sci 2003:44:4753-61.

14 Nichols KK, Nichols JJ, Mitchell GL. The lack of association between signs and symptoms in patients with dry eye disease. Cornea 2004;23:762-70.

15 Holland EJ, Luchs J, Karpecki PM, et al. Lifitegrast for the treatment of dry eye disease: results of a phase III, randomized, double-masked, placebo-controlled trial (OPUS-3). Ophthalmology 2017;124:53-60.

16 Sheppard JD, Torkildsen GL, Lonsdale JD, et al. Lifitegrast ophthalmic solution $5.0 \%$ for treatment of dry eye disease: results of the OPUS-1 phase 3 study. Ophthalmology 2014;121:475-83.

17 Tauber J, Karpecki P, Latkany R, et al. Lifitegrast ophthalmic solution $5.0 \%$ versus placebo for treatment of dry eye disease: results of the randomized phase III OPUS-2 study. Ophthalmology 2015;122:2423-31.

18 Petrillo J, Bressler NM, Lamoureux E, et al. Development of a new Rasch-based scoring algorithm for the National Eye Institute Visual Functioning Questionnaire to improve its interpretability. Health Qual Life Outcomes 2017;15:157.

19 Le Q, Zhou X, Ge L, et al. Impact of dry eye syndrome on visionrelated quality of life in a non-clinic-based general population. BMC Ophthalmol 2012;12:22.

20 Paulsen AJ, Cruickshanks KJ, Fischer ME, et al. Dry eye in the Beaver Dam Offspring Study: prevalence, risk factors, and healthrelated quality of life. Am J Ophthalmol 2014;157:799-806.

21 Herdman M, Gudex C, Lloyd A, et al. Development and preliminary testing of the new five-level version of EQ-5D (EQ-5D-5L). Qual Life Res 2011;20:1727-36.

22 Johnson ME, Murphy PJ. Measurement of ocular surface irritation on a linear interval scale with the ocular comfort index. Invest Ophthalmol Vis Sci 2007;48:4451-8.

23 Reilly MC, Zbrozek AS, Dukes EM. The validity and reproducibility of a work productivity and activity impairment instrument. Pharmacoeconomics 1993;4:353-65.

24 Abetz L, Rajagopalan K, Mertzanis P, et al. Development and validation of the impact of dry eye on everyday life (IDEEL) questionnaire, a patient-reported outcomes (PRO) measure for the assessment of the burden of dry eye on patients. Health Qual Life Outcomes 2011;9:111.

25 Chalmers RL, Begley CG, Caffery B. Validation of the 5-Item dry eye questionnaire (DEQ-5): discrimination across self-assessed severity and aqueous tear deficient dry eye diagnoses. Cont Lens Anterior Eye 2010;33:55-60.

26 Ngo W, Situ P, Keir N, et al. Psychometric properties and validation of the Standard Patient Evaluation of Eye Dryness questionnaire. Cornea 2013;32:1204-10. 